\title{
Membrane function: Its relationship to intestinal absorption and malabsorption
}

JB MEDDINGS, MD

ABSTRACT: It is now clear that both chemical composition and physical properties of the intestinal microvillus membrane are critically important in determining its transport function. Recent advances in this field have been made in research laboratories around the world, and it is important that such data be put in a clinical perspective. The concept of membrane fluidity and the ability to regulate this membrane parameter either pharmacologically or by diet is now an important concept in systemic and local gastrointestinal disease. This review gives an overview of membrane physical properties, how they are measured, and their relationship to nutrient absorption in the intestinal tract. It is not intended to be all inclusive and expressly attempts to make these concepts simple. Can J Gastroenterol $1990 ; 4(1): 39-46$

Key Words: Glucose, Intestinal absorption, Lipids, Membrane fluidity

Fonction de la muqueuse: Sa relation avec l'absorption et la malabsorption intestinales

RESUME: Il est maintenant clair que la composition chimique et les propriétés physiques des microvillosités de la muqueuse intestinale sont toutes deux d'une importance déterminante dans la fonction de transport de cette muqueuse. Des progrès récents ont été réalisés dans les laboratoires de recherche du monde entier et il est important que les données ainsi recueillies soient placées dans une perspective clinique. Le concept de la fluidité de la muqueuse et la capacité de régler ce paramètre soit par des moyens pharmaceutiques soit par le régime alimentaire, est désormais un concept important dans les affections gastrointestinales locales et systémiques. Ce compte rendu passe en revue les propriétés physiques de la membrane, examine comment elles sont mesurées et comment elles sont reliées à l'absorption de nutriments par l'intestin. Il ne prétend pas être exhaustif et tente surtout de clarifier ces concepts.

Gastrointestinal Research Group, Calgary, Alberta

Correspondence and reprints: Dr JB Meddings. Department of Internal Medicine,

Health Sciences Centre, 3330 Hospital Drive NW, University of Calgary, Calgary.

Alberta T2N 4N1. Telephone (403) 220-6883

Received for publication June 12, 1989. Accepted October 24, 1989
T HE STUDY OF CELL MEMBRANES HAS become popular once again in the 1980s. This has resulted in large part from the abandonment of the concept that the plasma membrane simply separates the inside and outside of the cell. Plasma membranes are now recognized to be the site of furious activity, directing the interaction between the cell and its external environment. Although this is true of all cells, knowledge concerning recent research in this area is of special interest to gastroenterologists as it is directly tied to a fundamental appreciation of the process of intestinal absorption and, hence, malabsorption.

The process of intestinal nutrient absorption has fascinated generations of physiologists, biochemists and clinicians alike. However, despite volumes of published papers and never-ending textbook chapters, there are glaring gaps in our understanding of how nutrients ultimately cross the intestinal microvillus membrane and gain access to the body. The importance of this to the clinician is embodied in one of Murphy's famous laws "whenever something can go wrong, it will". Historically, there have been examples of malabsorption involving most, if not all, of the steps involved in nutrient absorption. Despite this, from a clin- 


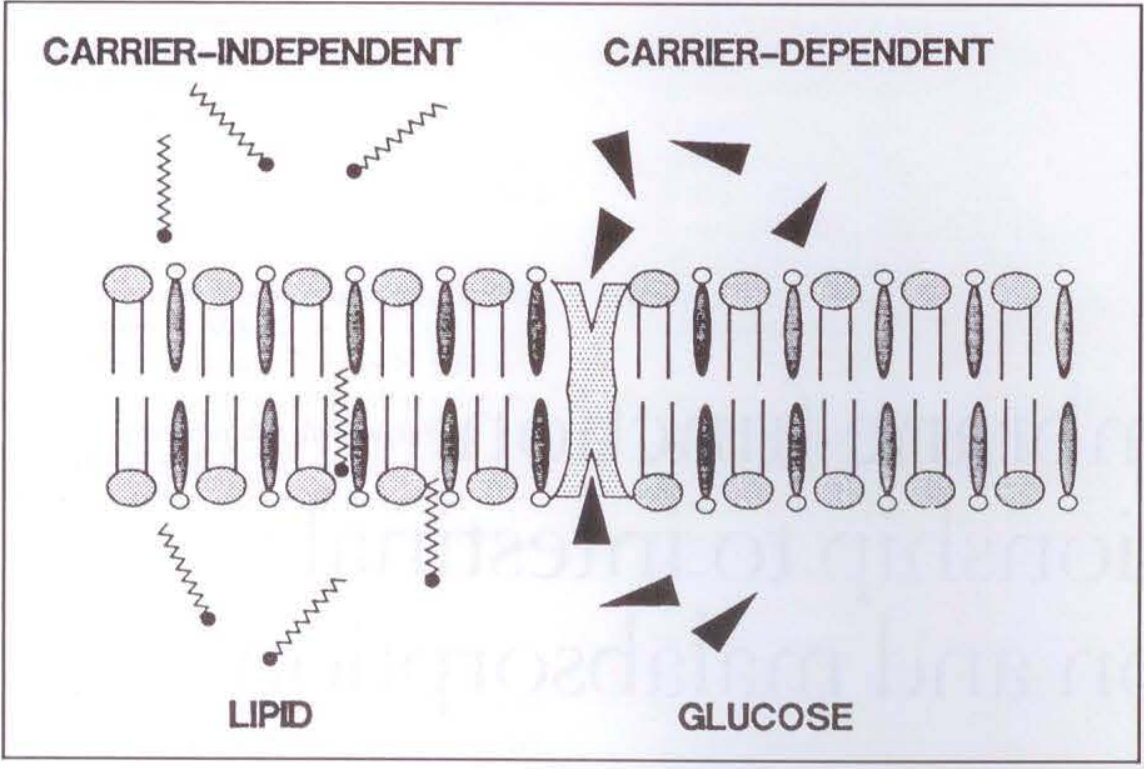

Figure 1) Mechanisms of transport across the microvillus membrane. A stylized membrane formed from cholesterol and phospholipid is depicted. A transmembrane protein transporter is in the centre of the field and on the left fatty acid permeation across the bilayer is illustrated. On the right the inability of glucose to cross the membrane directly is demonstrated. The mechanism by which the glucose carrier facilitates transmembrane movement is not entirely elucidated at present

ical perspective it remains equally clear that in many cases the cause of malabsorption is obscure and the methods used to deal with it are best described as limited. Thus, in order to keep abreast of future clinical developments it is wise to have a basic understanding of the current research efforts aimed at the fundamental principles of membrane nutrient transport.

This article will outline some recent advances in knowledge concerning the mechanism by which both lipids and more water-soluble nutrients cross the intestinal microvillus membrane. Specifically, this discussion will focus on the microvillus membrane and how its chemical structure and physical properties may combine to modulate nutrient absorption. Although this may initially appear esoteric to some, in the near future the information now in research circles may have important clinical applications.

The approach to this discussion will be first to describe, in general terms, what is known about membrane nutrient transport and relate this to the structure of the microvillus membrane. Since membrane biophysical properties appear to be of importance in the regulation of nutrient absorption, what is meant when a researcher refers to 'membrane fluidity' will be described and an attempt made to point out the relationship between this artificial measurement and the natural process of nutrient absorption. Finally, these concepts will be summarized and their significance to the clinical problems of malabsorption and its treatment noted

\section{INTESTINAL NUTRIENT TRANSPORT}

In its simplest form we can picture the small intestine as a single large membrane extending from the duodenum proximally to the cecum distally. Obviously, this formulation ignores the intercellular spaces through which much absorption occurs; however, for the sake of simplicity this contribution to absorption will not be considered here. In order for any nutrient to gain access to the body it must first cross the microvillus membrane. Herein lies the importance of understanding membrane structure and function. The microvillus membrane is simply a plasma membrane specially adapted for the efficient movement of nutrients from the intestinal lumen to the cytoplasm of the enterocyte. Importantly, it is now recognized that these adaptations involve not only protein transporters (or carriers) but also the lipid environment in which they are embedded.

Two major forms of membrane trans- port exist, generally termed the carrierdependent and the carrier-independent routes (Figure 1) (1). The evolution of these probably stemmed from the existence of two important classes of biological nutrients: those that are water soluble and those which are not. The latter generally fall into the class of compounds labelled lipids and represent a significant component of the daily diet. Since lipids are more or less soluble in organic solvents, they can permeate directly through most biological membranes, including the intestinal microvillus membrane which in its simplest form approximates a slightly polar organic solvent (2). This allows these nutrients a distinct advantage over their counterparts, the water-soluble nutrients. Since lipids do not require the assistance of a membrane transporter they enjoy the luxury of an almost infinite surface area for absorption. Consequently the amount of lipid absorbed depends upon only two factors: the existing concentration gradient across the membrane and the intrinsic permeability properties of the microvillus membrane $(3,4)$.

However, when a nutrient such as glucose approaches the membrane it is in a much more difficult predicament. Being highly water soluble and, therefore, insoluble in the nonpolar interior of the bilayer, glucose cannot gain access directly to the interior of the enterocyte. Since many of the nutrients we require fall into this class of water-soluble compounds we have evolved a diverse class of carrier proteins that facilitate the movement of such molecules across cell membranes using a bewildering variety of steps. In general these carrier molecules are transmembrane proteins, or groups of proteins, that may or may not be directly linked to a metabolic energy source such as ATP. Since it is a timeand energy-consuming process to manufacture such carriers there are generally a limited number of such sites, carefully quantitated over the course of evolution to approximate the number required to deal with a certain amount of nutrient. Therefore, for nutrients using the carrier-dependent route, only a severely limited surface area of membrane is available for absorption. The net result is that the amount of nutrient 
absorbed, in contrast to the carrierindependent route, is not linearly related to increasing concentration. A finite number of carriers implies that all available carriers can be saturated if enough nutrient is ingested. This creates a problem for animals, including humans, for whom a variable amount of dietary nutrient is the rule. How can the cell ensure that no metabolically expensive transporters go to waste and still absorb all available nutrients?

One possible solution would allow a small percentage of ingested nutrient to be ignored when dietary nutrients are plentiful and support only enough carriers to cope with the average meal. This solution has the advantage that the animal need not carry a large number of underused carriers. However, the optimal solution would allow for some sensing of the dietary nutrient load and a corresponding increase or decrease in activity of the required carriers.

At first glance it would seem that such a system could be accomplished most simply by regulating the factor not yet considered: time. Under conditions in whith an increased dietary load is ingested, a simple delay in intestinal transit would allow a saturated transport system to operate for a longer time. Although appealing and applicable to many conditions this solution does not cover all eventualities. It is quite easy to imagine situations in which the amount of one nutrient falls and another increases, making intestinal transit time a poor choice as the regulatory step. It would seem that a well designed system would incorporate multiple checks and balances that include the ability to change the activity of individual transport processes.

An initial step would involve up or down regulation of the synthesis of different transporters. This has now been convincingly demonstrated to occur in a variety of experimental systems. The prolonged feeding of a single nutrient in high concentrations induces the appearance of a greater number of transporters specific for that nutrient (5). However, such a mechanism does not allow for minute-to-minute adjustments in transporter activity depending on luminal or serosal conditions, since the syn- thesis of new proteins is time consuming. The disturbing fact is that such regulation does indeed occur. Acute perfusion of the intestinal lumen with high glucose concentrations immediately up regulates the functional activity of the glucose transport system (6). Furthermore, acute hyperglycemia, induced by glucose infusion, produces a similar result in the experimental animal (7). Thus, acute exposure to glucose, at either the luminal or serosal surface can trigger mechanisms resulting in rapid adaptation that allow the cell to make better use of this nutrient.

The mechanisms by which such rapid regulation of transport processes occur are presently being unravelled, and although multiple mechanisms probably exist, it is now clear that the physical state of the microvillus membrane may have a major impact on nutrient absorption. Within the last 10 years the concept of biological membranes has undergone a dramatic shift. No longer are they viewed as static, unchanging barriers that simply serve to support cellular shape. The older view of a 'picket fence' membrane has gradually given way to one in which the membrane is seen as a dynamic structure. Within the microvillus membrane lipid molecules are in constant motion, rapidly changing both position and form on a minute-to-minute basis. It is appropriate at this point to consider a simplified view of membrane structure.

\section{MEMBRANE STRUCTURE AND PHYSICAL PROPERTIES}

Since the original proposal by Singer and Nicholson (8) of the fluid-mosaic model for membrane structure, it has become accepted that most biological membranes are formed from two hemileaflets (Figure 1). Each hemileaflet contains lipid and protein; the protein component can span both leaflets, as in the case of membrane transporters. However, it is the lipid composition of biological membranes that is responsible for the diversity of membrane structure that is now being appreciated. From one perspective only two lipid types form the vast majority of membrane lipids: cholesterol and phospholipids. However, the subclasses of phospholipids are extensive. The chemical structure of a typical phospholipid is shown in Figure 2. The head-



Figure 2) Structure of a typical phospholipid. Phospholipids are formed from a glycerol backbone to which is attached a headgroup via a phosphate linkage. This group is illustrated as R3 and can be one of many groups. Six are listed in the figure. The diversity of phospholipid structure becomes apparent when the fatty acyl groups (RI and R2) are considered. There are many different fatty acids that can combine to produce individual phospholipid species. Not all of the possibilities are listed. In general. fatty acids in the RI position are saturated while those substituting for $R 2$ are mono-or polyunsaturated 
group of these molecules (R3) defines the class of the phospholipid; most membranes contain at least five major classes. The incredible diversity in membrane lipid composition becomes apparent when the phospholipid acyl chains are examined. A typical phospholipid has two fatty acids attached to the glycerol base, generally one saturated (Rl) and one unsaturated (R2) acyl chain. However, the number of possible choices for each fatty acid is huge and the resulting combinations seemingly endless, thereby affording a high degree of flexibility to the system.

It is also important to recognize that once a membrane is formed its lipid structure is not etched in stone. On a minute-to-minute basis changes can occur, primarily in the phospholipid species with alterations of both headgroups and fatty acyl chains. Such changes are mediated by a variety of enzyme systems located in the plasma membranes of most cell types. Only a few of the stimuli that precipitate these changes are known, but the fact remains that membrane lipids can be altered over a rapid timescale, the resultant change affecting membrane function. The major impediments to further research in this area are twofold. First, it is difficult to isolate and characterize membrane lipids with any degree of precision. Second, the sheer diversity of membrane lipid types makes a meaningful interpretation of such data almost impossible. Following almost any stimulus a given membrane may have numerous lipid species either increase or decrease in number. An investigator can almost select the number of significant alterations he or she wishes to find by performing the lipid analysis either superficially or in great depth. The more sophisticated the analysis, the greater the number of differences that can be identified. The question then becomes whether or not small yet statistically significant changes have functional meaning. This problem has led many investigators to seek tools that provide an overall assessment of membrane lipid structure which might help to evaluate the significance of many small compositional changes. One of the leading candidates is the measurement of 'membrane fluidity'.

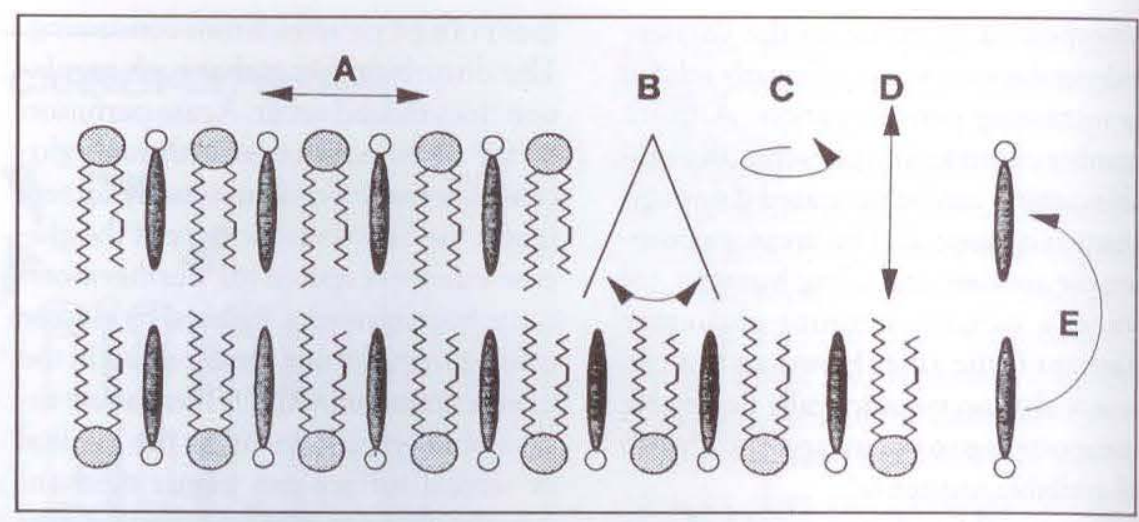

Figure 3) Types of motion within biological membranes. Several types of molecular motion are depicted. A Lateral diffusion; B Wobble; C Rotation; D Bobbing; E Flip-flop

A cornerstone in the model of biological membranes is molecular motion: membranes are dynamic. The degree of motion in a particular membrane is defined by that membrane's chemical structure and, therefore, if 'membrane motion' could be measured one would have a global measurement of membrane structure. The major problem with this approach is the definition of membrane motion. Numerous types of movement are possible in a membrane (Figure 3). Molecules can move laterally within the plane of the membrane, rotate, wobble, 'bob' or even flip from one leaflet to the other. Therefore, to truly assess motional freedom in a given membrane, each type of motion should be examined. This becomes a timeconsuming task but is considerably simpler and more meaningful than the alternative - complicated lipid analyses. Furthermore, to correlate alterations in transport function with changes in the membrane there are theoretical arguments suggesting that transport rates should in fact be determined by the physical state of the membrane rather than by specific lipid composition itself (9). Thus, it makes sense to measure the fluidity or rigidity of a particular membrane and assess whether this property of cellular membranes is regulated.

There are very simple methods, both in theory and practice, now available to measure this function of biological membranes. Ideally, one would like to label a particular molecule, locate its exact position at time zero and a short time later measure how far it has moved. In practice it is usually not possible to use an endogenous molecule; rather an exogenous molecule is added and its motion carefully measured. It is assumed that the motion of this molecule approximates that of endogenous membrane lipids. The techniques most prevalent today use fluorescent lipid molecules as tracers to assess three types of motion: the amount of wobble allowed in a membrane, the rotational freedom of a probe, and the amount of lateral diffusion that can occur within the plane of the bilayer. These techniques can be further extended to provide information at various depths within the bilayer and many laboratories, including the author's, routinely quantitate rotational freedom at various depths within the bilayer. These estimates of motional freedom are relatively simple to obtain and provide an estimate of membrane fluidity. Although not strictly correct, for the purpose of this review 'fluid' can be thought of as the inverse of viscous. Thus, a fluid membrane allows more movement of any type than a rigid or viscous membrane. In most instances all three types of motion are in agreement; however, there is no a priori reason why lateral diffusion cannot decrease while rotational freedom increases. A final caveat must be kept in mind. When fluorescent probes are introduced into a membrane sample they enter the bilayer randomly. Therefore, the signal obtained represents an overall average from all regions that the probes have entered. Thus, if a large change has occurred in a localized region of the membrane, for instance in the lipid immediately adjacent to the glucose transporter, this change might be 
missed since it represents a small fraction of the whole signal.

Given that an estimate can be made of the fluidity of a particular membrane, the next question is the relation of this to membrane function. One area of particular interest is nutrient transport across the intestinal microvillus membrane and the following discussion will be limited to this area. At present only a handful of laboratories have examined these questions and, therefore, the following data leans heavily upon work that has emerged from only a few groups.

\section{RELATIONSHIP BETWEEN MEMBRANE TRANSPORT AND FLUIDITY}

Along the length of the small intestine, at both macro- and microscopic levels, there are a variety of 'axes' over which transport function appears to vary. These include the jejunal-ileal axis and the crypt-villus tip axis. Fat and glucose absorption are typically complete by the end of the jejunum, while bile acid and $B_{12}$ absorption take place in the ileum. Villus tip enterocytes are absorptive, while erypt enterocytes are said to be predominantly secretory and not to absorb nutrients such as glucose. If a relationship exists between the physical properties of the microvillus membrane and its transport activity one would, therefore, expect the physical properties of this membrane to vary over these axes.

When this is examined it is found to be true. In a number of species it now appears that the microvillus membrane becomes progressively more rigid as cells are sampled from jejunum to ileum $(10,11)$. This is true for all types of motion within the bilayer that have been examined. Furthermore, over the lifespan of several experimental animals the microvillus membrane becomes more rigid with age (12). Although only a small amount of data relating to transport function in aged animals is available, an analogous situation occurs along the cryptvillus axis. Following its 'birth' in the intestinal crypts the enterocyte migrates up the villus and after reaching the villus tip is sloughed into the intestinal stream. Techniques are now available to isolate sequential fractions of these cells and compare mature villus tip cells to imma- ture crypt cells. It is now clear in two species that the microvillus membrane becomes more rigid as the enterocyte matures and moves up the crypt-villus axis $(13,14)$. Thus, in many situations for which membrane transport function differs there are also differences in membrane physical properties.

\section{LIPID ABSORPTION}

It is not enough simply to correlate one variable with another to prove a relationship. For instance, even though ileal microvillus membrane is more rigid than jejunal microvillus membrane is this the reason that lipid absorption is less efficient in the ileum than in the jejunum? Many factors regulate the rate of lipid absorption. Since lipids simply permeate through the microvillus membrane it seems intuitive that the rate of permeation must depend upon some physical characteristics of the membrane. It has been proposed, from mathematical arguments, that the rate-limiting step for transmembrane lipid permeation should be the density of membrane lipids in the outer third of the bilayer, just beneath the phospholipid headgroups. Physical properties can now be measured at different depths in the bilayer to answer this question. The measurement of rates of fatty acid permeation across jejunal and ileal microvillus membranes reveals that the lipid permeability properties of jejunal microvillus membrane far exceed those of ileal microvillus membrane. Ileal microvillus membrane is generally more rigid than jejunal microvillus membrane. This difference is localized, in large part, to the superficial regions of the membrane (11). Thus, these experiments appear to bear out the proposed theoretical relationship between membrane physical properties and lipid permeability. However, once again these are simply correlative studies which do not provide firm evidence to support the hypothesis. In order to test this hypothesis one would need to alter the physical properties experimentally within this region of the bilayer, and demonstrate altered membrane lipid permeability.

The primary determinant of membrane fluidity in the outer region of the bilayer is cholesterol. This molecule has a single polar hydroxyl group that aligns itself at the membrane water interface. Immediately below this group are the four carbon rings that form the basis of all sterols. This region of the molecule is highly rigid and forms strong hydrophobic interactions with neighbouring phospholipid side chains. In order to alter physical properties in the outer third of the bilayer, the cholesterol ring structure must be modified. Fortunately, pharmaceutical companies have recently developed a variety of compounds which interfere with the biosynthesis of cholesterol and force the production of alternate sterols having different ring structures. Using one of these inhibitors it is possible to replace microvillus membrane cholesterol completely, in vivo, with a precursor of cholesterol. Animals treated with this agent appear grossly normal and develop much like control animals, thereby excluding gross derangements in intestinal transport function. However, as expected their jejunal microvillus membrane is significantly more rigid than that of control animals. Furthermore, this difference is limited to the outer regions of the membrane, the region implicated in the control of lipid permeation rates. These animals could thus be used to test the hypothesis outlined above. When lipid permeation rates were measured in these animals the more rigid microvillus membrane of the treated animals had a much lower lipid permeability than that of the controls, thereby confirming the prediction (15).

It now seems safe to conclude that at least one more regulatory step in the rate of lipid absorption is the physical state of the outer bilayer of the microvillus membrane. From a physiological and clinical perspective it is important to ask whether the body regulates lipid permeation rates by altering the physical properties of the microvillus membrane in vivo. If this were the case one would anticipate that under conditions of a high dietary lipid content the microvillus membrane would be kept fairly fluid and as the dietary content of lipid decreased the membrane would become more rigid. This is exactly the scenario that occurs when an infant drinking breast milk weans and begins to ingest solid food. Breast milk has most of its caloric content as lipid; after weaning the majority 
of calories are found in carbohydrate. It was, therefore, of interest to study the development of the intestine in the experimental animal over this time course (16).

In the suckling rat the jejunal microvillus membrane is highly permeable to lipid and this permeability rapidly decreased at the time of weaning. From a structural viewpoint this coincided with a remarkable change in the lipid composition of the microvillus membrane. Major alterations were seen in the amount of membrane cholesterol, the type of membrane phospholipids and their fatty acyl composition. In fact the compositional changes are so numerous that they become confusing. However, when the physical properties of the microcrovillus membrane were examined over the same time course this apparently confusing situation became more clear. During the suckling period the jejunal microvillus membrane is extremely fluid, especially in its outer regions, and within days of weaning to a solid diet it becomes dramatically more rigid. These alterations occurred over the same time course as the observed changes in membrane lipid permeability.

Therefore, it now appears that the degree of membrane fluidity can not only regulate the lipid transport properties of the microvillus membrane, but also allow the animal, and presumably humans, to adapt to alterations in dietary nutrients under certain conditions.

\section{CARRIER-DEPENDENT NUTRIENT ABSORPTION}

Since carrier-independent transport processes occur directly through the substance of the bilayer, it is relatively simple to convince oneself that the physical properties of the bilayer will be important in determining rates of transport. Carrier-dependent processes require the intervention of a protein carrier and thus it is not immediately apparent that membrane lipids will affect the function of this protein. However, still incompletely appreciated is the exact mechanism by which a carrier protein binds to its substrate and moves glucose, for instance, across the membrane. It seems reasonable that this movement requires a change in conformation of the protein, and it is entirely con- ceivable that the degree of conformational change may be limited by the fluidity of the microvillus membrane. Most carrier-dependent transport systems are characterized on the basis of two parameters; the affinity of the transporter for its substrate (inversely proportional to the Michaelis-Menten constant $\left[\mathrm{K}_{\mathrm{m}}\right]$ of the system) and the maximal transport velocity $\left(\mathrm{J}_{\max }\right)$. The latter is determined by two further parameters: the number of transporters and their turnover number, which is a reflection of how rapidly the transporter can move substrate from one side of the membrane to the other and return to its starting position.

In the case of the glucose transporter located in the microvillus membrane, it is now known that the step initiating glucose movement is the binding of a sodium ion to its active site on the transporter (17). The protein has been shown to undergo a conformational change associated with this binding that presumably exposes the glucose binding site, since the affinity for glucose binding increases dramatically with prior sodium binding. What occurs following binding of glucose at the exterior of the membrane is unclear. Somehow glucose bound to the transporter on the outside of the membrane moves to the inner surface of the microvillus membrane and is released. The exact steps that accompany this translocation are unknown. However, it is likely that they involve further conformational changes in the protein. From a theoretical perspective, if the degree of conformational change limited the physical state of membrane lipids surrounding the protein then the degree of membrane fluidity would affect both the $J_{\max }$ and the $K_{m}$ of the glucose transport process.

Does this occur with the intestinal sodium-dependent glucose transporter? There are several studies that have examined the function of this transport system following alteration of the lipid content of the microvillus membrane, usually by dietary means (18-20). In many cases alterations in the activity of the transport system were observed, suggesting that this transporter might be sensitive to changes in the lipid environment. However, no clear demonstration of a relation- ship to membrane fluidity has emerged until recently.

Proceeding in a manner similar to that presented for lipid permeability, enterocytes from along the crypt-villus axis have recently been isolated and the kinetics of glucose transport across the microvillus membrane of these cells examined. It is quite clear that rates of glucose movement are dramatically reduced across crypt microvillus membrane compared to villus tip microvillus membrane. Furthermore, the differences in glucose transport rate involve both the maximal glucose transport rate as well as the affinity of the transporter for glucose. Although the crypt transporter has a much lower maximal transport velocity, it has an affinity for glucose that is almost twice as high as that seen in villus tip enterocytes (14). Remember that crypt microvillus membrane is far more fluid than its counterpart in the mature villus tip enterocyte. The question, therefore, becomes whether these differences can be accounted for by the difference in membrane lipid environment or whether the crypt transporter is fundamentally different from the one seen in the villus tip.

In answering this question the first consideration is the affinity of the transport system. It has now been shown that the $\mathrm{K}_{\mathrm{m}}$ of the glucose transport system for glucose is close to $100 \mu \mathrm{M}$. Furthermore, this is dependent upon the concentration of sodium ions in the medium. If sodium is gradually removed, the affinity of the transporter for glucose falls and the $\mathrm{K}_{\mathrm{m}}$ increases to something in the order of $200 \mu \mathrm{M}$. In crypt cells the $\mathrm{K}_{\mathrm{m}}$ of the transporter is close to $100 \mu \mathrm{M}$ while in the villus tip cells it approximates 200 $\mu \mathrm{M}$. One explanation for these data is that the conformational change induced by sodium binding can occur only in the fluid environment of the crypt microvillus membrane; the villus tip microvillus membrane is too rigid to allow this degree of movement. To test this hypothesis the $K_{m}$ of the crypt transport system has been examined at various sodium ion concentrations. At low concentrations the $\mathrm{K}_{\mathrm{m}}$ for the system does indeed increase to $200 \mu \mathrm{M}$, suggesting that this is the same transporter as found in villus tip cells, simply in a different lipid 
environment (14). Further support for this hypothesis came from experiments where villus tip microvillus membrane was artificially made as fluid as crypt microvillus membrane and the effect of this manipulation upon glucose transport kinetics examined. Using benzyl alcohol as a membrane fluidizer it has been demonstrated that fluidization of villus tip microvillus membrane has two major effects on the kinetics of glucose transport. First, as expected, it increases the affinity of the transport system for glucose. The $\mathrm{K}_{\mathrm{m}}$ for the transport process decreases from 200 to $100 \mu \mathrm{M}$, providing further support for the argument that it is the physical state of the membrane lipids that determines the degree of conformational change that can occur when sodium binds. Second, fluidizing villus tip microvillus membrane profoundly reduces the maximal velocity of glucose transport $\left(\mathrm{J}_{\max }\right)$ to levels seen in native crypt microvillus membrane. Thus, it now appears that the physical properties of the microvillus membrane are important in the modulation of not only carrier-independent transport processes but also in one important example of carrier-dependent nutrient absorption.

\section{SUMMARY}

What does all this mean to the practising gastroenterologist? At the present time there may be more theory than practical implications. The important point is that compelling evidence now exists which suggests that the physical properties of the microvillus membrane can affect rates of nutrient transport. Such knowledge gives a better appreciation of the process of normal absorption and hopefully makes one consider the pathophysiology of malabsorption more carefully. One might find by looking at cases of idiopathic malabsorption more closely that some have defects in the control of microvillus membrane fluidity. Furthermore, the physical properties of the microvillus membrane can now be controlled to some extent. As discussed in a previous section there are pharmacological agents that produce demonstrable changes in membrane lipid composition, fluidity and transport function. By altering dietary patterns many investigators have been able to alter microvillus membrane lipid composition and transport function.

A multitude of questions stem from these observations that are important to the practice of medicine. It is still not understood why some patients hyperabsorb' certain sterols including cholesterol and plant sterols. The defect in these patients may occur at the level of the microvillus membrane and even if not it is at a level at which one can interfere therapeutically in the process of absorption. Furthermore, certain diseases, such as diabetes, result in gross derangements of membrane structure. A pertinent clinical problem commonly faced is the control of postprandial hyperglycemia in diabetics that is secondary to 'hyperabsorption' of luminal glucose. While numerous strategies have been proposed to control this problem, it would seem logical to direct therapy at the cause Recent work in experimental diabetes has demonstrated that the marked intestinal glucose absorption can be controlled by alteration of the structure of membrane lipids through dietary manipulation (18). Furthermore, in Western countries the dietary intake of cholesterol and saturated lipids is excessive. One of the problems associated with this is the high circulating concentration of low density lipoprotein (LDL) cholesterol and its associated high incidence of coronary artery disease. What is not generally appreciated is that the small intestine is a logical place to look for a solution.

Outside the liver the small intestine has the greatest number of LDL recep- tors in the body (21). This is probably due to the fact that the epithelium turns over rapidly and therefore requires a continuous source of cholesterol for the synthesis of new membrane. The enterocyte has three sources of cholesterol. First. dietary cholesterol enters across the microvillus membrane. Second, cholesterol can be synthesized intracellularly from acetate and third, the enterocyte can use LDL cholesterol absorbed across the basolateral membrane with the help of the LDL receptor. A significant reduction in plasma LDL cholesterol concentration could be achieved if the enterocyte could be stimulated to up regulate the number of these receptors, thereby increasing the amount of LDL cholesterol cleared from the plasma (22). From a theoretical perspective this could be achieved by inhibiting the synthesis of new cholesterol within the enterocyte and the absorption of dietary cholesterol. If one simply removes cholesterol from the diet the enterocytes' first choice is to increase synthesis of new cholesterol rather than LDL clearance. However, there are now available drugs such as lovastatin that potently inhibit the ability of cells to synthesize cholesterol. With the understanding of lipid permeability across the microvillus membrane outlined above it is possible to design maneuvres to in hibit cholesterol absorption. These coupled with the use of drugs such as lovastatin one could theoretically produce a situation which would dramatically reduce the serum LDL cholesterol concentration.

Thus there will soon be a variety of therapeutic options that can alter small intestinal transport function by directly affecting the lipid environment of the microvillus membrane. In order to use these efficiently and wisely it is important to have at least a passing knowledge of the interaction between membrane lipids and membrane transport processes.

\section{REFERENCES}

1. Macey RI. Mathematical models of membrane transport processes. In: Andreoli TE, Hoffman JF, Fanestil DD Schultz SG, eds. Physiology of Membrane Disorders. New York: Plenum. 1986:111-31.

2. Sallee VL. Dietschy JM. Determinants of intestinal mucosal uptake of short and medium chain fatty acids and alcohols. J Lipid Res 1973;14:475-84

3. Thomson ABR, Dietschy JM. Intestinal lipid absorption: Major extracellular and intracellular events. In: Johnson LR, ed. Physiology of the Gastrointestinal Tract New York: Raven Press, 1981:1147-220
4. Westergaard H. Dietschy JM. Delineation of the dimensions and permeability characteristics of the two major diffusion barriers to passive mucosal uptake in the rabbit intestine. J Clin Invest 1974:54:718-32

5. Ferraris RP, Kwan WW. Diamond ] Regulatory signals for intestinal amino 
acid transporters and peptidases. Am ] Physiol 1988:255:G151-7.

6. Csaky TZ. Fischer E. Intestinal sugar transport in experimental diabetes. Diabetes 1981:30:568-74.

7. Maenz DD, Cheeseman CI. Effect of hyperglycemia on D-glucose transport across the brush border and basolateral membrane of rat small intestine. Biochim Biophys Acta 1986;860:277-85.

8. Singer SJ, Nicholson GL. The fluid mosaic model of the structure of cell membranes. Science 1972:175:720-31.

9. Stein WD. In: Bonting SE, De Pont JJHHM, eds. Membrane Transport. New York: Elsevier, 1982:1-28.

10. Schacter D, Shinitzky M. Fluorescence polarization studies of rat intestinal microvillus membranes. $]$ Clin Invest 1977:59:536-48.

11. Meddings JB. Lipid permeability of rat jejunum and ileum: Correlation with physical properties of the microvillus membrane. Biochim Biophys Acta 1988;943:305-14.

12. Brasitus TA, Yeh K, Holt PR, Schachter D. Lipid fluidity and composition of intestinal microvillus membranes isolated from rats of different ages. Biochim Biophys Acta 1984;778:341-8.

13. Brasitus TA, Dudeja PK. Alterations in the physical state and composition of brush border membrane lipids of rat enterocytes during differentiation. Arch Biochem Biophys 1985;240:483-8.

14. Meddings JB, DeSouza D. Maturation of glucose transport along the crypt-villus axis of the rabbit. Gastroenterology 1989;96:A238

15. Meddings JB. Lipid permeability of the intestinal microvillus membrane may be modulated by membrane fluidity in the rat. Biochim Biophys Acta. (In press)

16. Meddings JB, Thiesen S. Development of rat jejunum: Lipid permeability, physical properties and chemical composition. Am J Physiol 1989:256:G931-40.

17. Peerce BE, Wright EM. Sodium-induced conformational changes in the glucose transporter of intestinal brush borders. J Biol Chem 1984;259: 14105-12.

18. Thomson ABR, Keelan M, Clandinin MT, Rajofte RV, Cheeseman CL, Walker $K$. Treatment of the enhanced intestinal uptake of glucose in diabetic rats with a polyunsaturated fatty acid diet. Biochim Biophys Acta 1987;905:426-34.

19. Thomson ABR, Keelan M, Clandinin MT, Walker K. A high linoleic acid diet diminishes enhanced intestinal uptake of sugars in diabetic rats. Am J Physiol 1987;252:G262-71.

20. Brasitus TA, Dudeja PK, Bolt MJ, Sitrin MD, Baum C. Dietary triacylglycerol modulates sodium-dependent Dglucose transport, fluidity and fatty acid composition of rat small intestinal brush-border membrane. Biochim Biophys Acta 1989: 979:177-86,

21. Spady DK, Meddings JB, Dietschy JM. Kinetic constants for receptor dependent and receptor independent low density lipoprotein transport in the tissues of the rat and hamster. J Clin Invest 1986;77:1474-81

22. Meddings JB, Dietschy JM. Regulation of plasma low density lipoprotein cholesterol levels: Interpretation of LDL turnover data in man. Circulation 1986;74:805-14. 


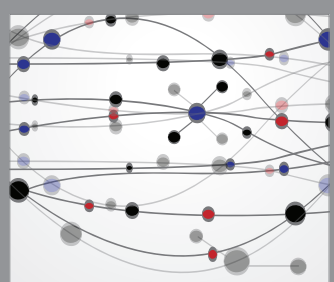

The Scientific World Journal
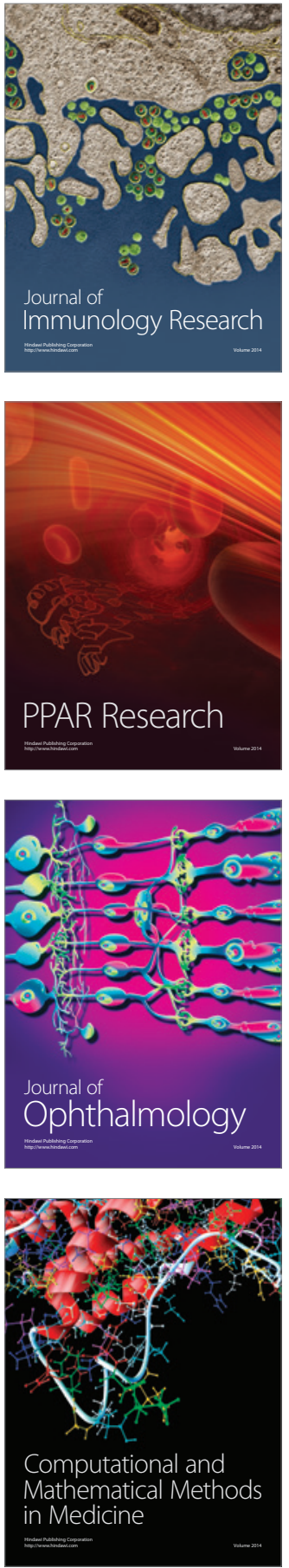

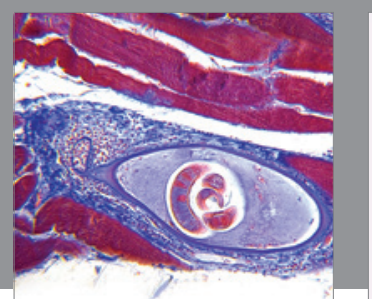

Gastroenterology Research and Practice

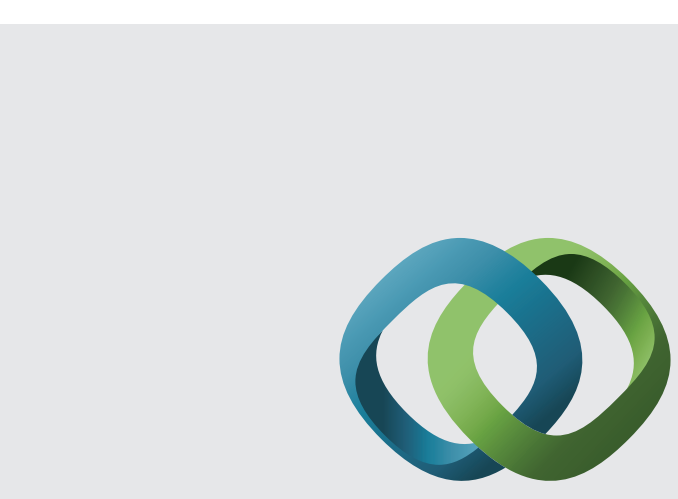

\section{Hindawi}

Submit your manuscripts at

http://www.hindawi.com
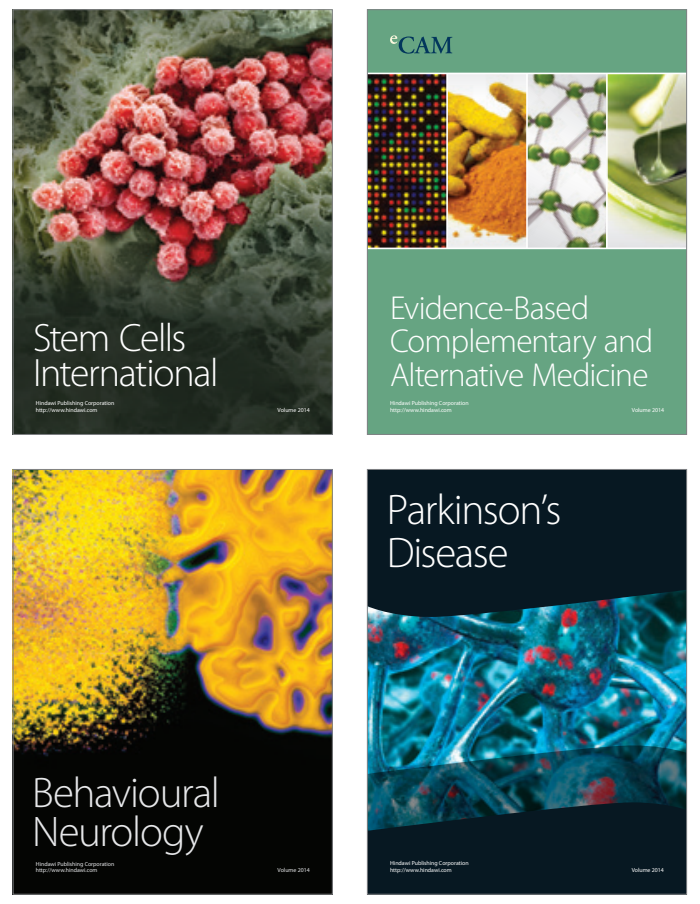
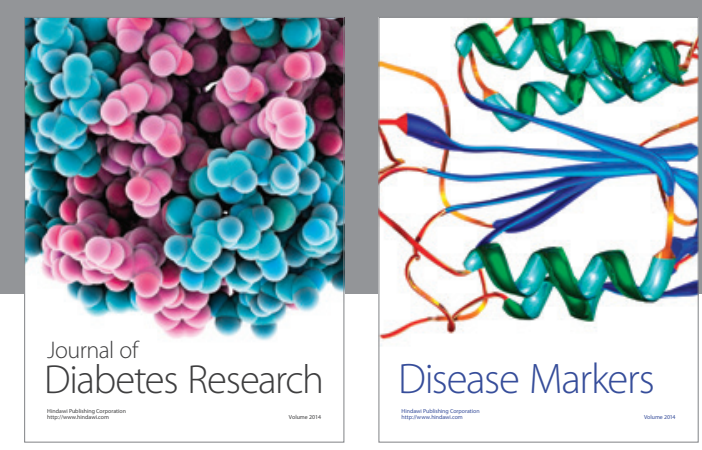

Disease Markers
